3

28

29

30

31

\title{
Molecular Signatures for Vaccine Development
}

\author{
J. Maertzdorf*, S.H.E. Kaufmann*, J. Weiner 3rd
}

Department of Immunology, Max Planck Institute for Infection Biology, Berlin, Germany

*Correspondence: Stefan Kaufmann, kaufmann@mpiib-berlin.mpg.de and Jeroen Maertzdorf, maertzdorf@mpiib-berlin.mpg.de: Max Planck Institute for Infection Biology, Department of

Immunology. Charitéplatz 1, D-10117 Berlin, Germany. 


\section{Abstract}

35 The immune system has evolved complex and specialized mechanisms to mount specific defense 36 responses against the various types of pathogens it encounters. For the development of new vaccines, it 37 is crucial to gain a better understanding of what these mechanisms are and how they work. The field of 38 vaccinology has adopted high-throughput profiling techniques to gain more detailed insights into the various immune responses elicited by different vaccines and natural infections. From all detailed

40 transcriptional profiles generated today, a general picture of immunological responses emerges. First, 41 almost every type of vaccine induces an early interferon-dominated signature. Second, different vaccine 42 formulations induce distinct transcriptional signatures, representing the highly specialized defense 43 mechanisms that must cope with the different pathogens and insults they cause. Transcriptional 44 profiling has shifted its attention towards early molecular signatures, with a growing awareness that 45 early innate responses are likely critical instructors for the development of adaptive immunity at later 46 time points.

47

48

49

50

51

52

53

54

55

\section{Keywords}

57 Signatures

58 Vaccination

59 Defense response 


\section{Introduction}

High-throughput transcriptional profiling studies are increasingly applied for detailed insights into the host response evoked by vaccines. The ultimate goal of such profiling studies is the identification of molecular biomarkers and signatures that can predict vaccine efficacy. Although such studies may capture the overall host response to vaccination in a single sketch, analysis of the complex data generated can be challenging. Key issues of study design include sample type and size, timing of sampling, data analysis, non-specific effects and impact of gender and age on outcome of vaccination [1]. A plethora of transcriptional profiling studies have been published over the past decade describing vaccine- or pathogen-related signatures both in vivo and in vitro. A number of these studies have already been reviewed by Wang et al. [2]. Here we will focus on most relevant, and most recently described, signatures.

\section{Signatures of natural infection}

The immune system has evolved complex and specialized mechanisms to counteract different pathogens with unique defense responses (see Box 1 for a short list of the key components of the host immune system that drive defense responses against pathogens). Many natural infections evoke potent immune responses, which effectively eliminate the pathogen and often induce long-lasting protection against reinfection. For these infections, understanding the mechanisms underlying protection against natural infection can provide valid information for vaccine design. Therefore, a closer look at these molecular signatures evoked by natural infection can be highly informative. This, however, is not true for all infections, since several pathogens are capable of manipulating the immune system. In these instances, vaccines need to be designed that induce a more efficient immune response than natural infection.

One intriguing study is the combined approach of Jenner et al. [3]. This systematic comparison collated and analyzed published transcriptional profiling datasets from 32 studies that involved 77 different host-pathogen interactions. Common host signatures induced in different cell types and in response to multiple pathogen species are likely to represent a general "alarm signal" of infection. The most strongly and consistently upregulated genes are almost exclusively pro-inflammatory mediators, including interferon (IFN)-related genes. Other shared host response genes are associated with lymphocyte activation, antigen presentation, cell adhesion and tissue invasion.

Transcriptional host signatures common to many host-pathogen interactions are mediated by a shared number of transcriptional regulators and cytoplasmic signal transducers that activate the immune response. Similarly, a "healthy" immune response also activates negative feedback mechanisms. It is thus not surprising that immune response-limiting genes and anti-apoptotic genes are represented as well in the shared host response signatures [3]. Yet, defense responses to distinct pathogens require preferential induction of genes in distinct cells, mediated by specific host factors. Combined, analysis of the different host-pathogen interactions suggests that the host response consists of a spectrum of 
transcriptional programs that form unique and specific combinations, involving numerous transcriptional regulators [3].

Professional antigen presenting cells (APCs), like dendritic cells (DCs), respond in a distinct manner, depending on their phenotype and the nature of stimulation. In an in vitro setting, DCs generated in the presence of interleukin (IL)-4 respond more strongly to influenza virus and Salmonella enterica (SE) infection than DCs differentiated with IFN $\alpha$ (IFNa DCs). However, response magnitude to Staphylococcus aureus (SA) is similar for both DC phenotypes [4]. Antiviral responses comprise various IFN-regulated genes, whereas early bacterial responses are primarily associated with signatures of proinflammatory molecules, and late bacterial responses are strongly dominated by genes involved in antigen presentation and maturation of APCs. ligands, cytokines and ligands for cytoplasmic receptors, induce different transcriptional signatures. TLR7 agonists (like single-stranded nucleotides), for example, induce signatures in IFN $\alpha$ DCs that closely correlate to those induced by influenza virus. SE shows induced signatures that best match with lipopolysaccharide (LPS) (i.e., a gram-negative bacterial cell wall component that acts as TLR4) and with responses induced by TLR7/TLR8-ligand [4]. Such diverse responses to inflammatory stimuli by phenotypically distinct cells emphasize that the experimental setup in high-throughput profiling studies can profoundly affect the outcome of the host response. Cellular signatures evoked by stimulation with different vaccines in the same study will be described in the next section.

\section{Signatures of vaccination}

122 In recent years, high-throughput transcriptional signatures have been investigated to study immune responses induced by existing and candidate vaccines. These studies primarily focus on early induced innate immune responses, rather than the more traditional adaptive responses at later time points (e.g., antigen-specific $T$ cell function). Different vaccines induce distinct transcriptional profiles depending on pathogen type, adjuvant formulation and target cell type. Such distinct signatures illustrate the diverse functionality of different APCs in responding to distinct groups of pathogens. The above-mentioned study by Banchereau et al. combined infection of DCs with vaccine-induced in vitro and in vivo responses [4]. Several subsets of DCs, as well as monocytes, were stimulated with 13 commercially available vaccine preparations. The signatures observed show that inactivated viral vaccine responses are dominated by IFN responses, while inactivated bacterial vaccines induce a much broader response. In addition, bacterial vaccines are more potent activators of inflammasome functions, DC maturation and Janus kinase/signal transducer and activator of transcription (JAK-STAT) signaling. In an in vivo setting, inactivated influenza virus preparations induce transcriptional signatures that are most prominent within the first 2 days post-vaccination. These signatures are mainly associated with activated pathways involved in DC maturation and T cell activation. Interestingly, similar signatures are induced in symptomatic but not in asymptomatic influenza-infected individuals. These findings 
underline that signatures from natural infections mirror activation by homologous vaccines and thus can provide valid information for rational vaccine design.

\section{Influenza}

The most detailed studies of vaccines, in terms of systems biology approaches, are those against influenza and yellow fever. Influenza globally affects an estimated $5 \%-10 \%$ of adults and $20 \%-30 \%$ of children annually, with particular risk for certain groups of individuals [5]. Lorenzo et al [6] reviewed the immunobiology of influenza vaccines with a strong focus on profiles underlying symptomatic infections and signatures predictive of vaccine immunogenicity.

The two major vaccine formulations available for influenza are live attenuated influenza vaccines (LAIV) and trivalent inactivated influenza vaccines (TIV). In human vaccination studies, IFN signaling and antigen presentation are uniformly seen as major functional processes affected by influenza vaccination [7-10]. Nevertheless, these two formulations induce significantly different transcriptional signatures in humans [7;9;10]. LAIV induces a considerably higher number of regulated genes [7;10], where most changes in gene expression are induced in the plasmacytoid DC population. TIV induces higher virusspecific IgG-secreting plasmablasts, which relates to the observation that subjects vaccinated with this formulation display the most substantial gene regulation in myeloid DC and B cell populations [9]. Nakaya et al [9] has shown that response magnitude (in terms of antibody titers at later time points) induced by TIV correlates with gene expression signatures enriched for genes related to antibodysecreting B cells. Conversely, in low-responding individuals the expression response is over-represented by genes involved in T cell signaling pathways. The induction of virus-neutralizing antibodies can already be predicted based on gene expression patterns within 24 hours after immunization [8]. Early IFN signaling and antigen processing are more pronounced in high responders.

Such an early modular IFN signature has been confirmed in a study comparing influenza and pneumococcal vaccines [11]. Both vaccines induce genes related to increased apoptosis and lower T cell and protein synthesis activity. In contrast, the pneumococcal vaccine elicits a more pronounced inflammatory response.

\section{Yellow fever}

Yellow fever, an acute hemorrhagic disease transmitted by mosquitoes, causes an estimated 30,000 deaths globally each year, mostly in Africa [12]. Vaccines against yellow fever are available and induce potent protective response. The live attenuated vaccine YF17D induces an integrated immune response involving multiple arms of the immune system, which is preceded by a coordinated upregulation of transcription factors like STAT1, IRF7 and ETS2 [10;13;14]. Transcriptional changes are most prominent within the first week where increased expression of genes is related to IFN and innate antiviral responses, including complement and inflammasome. This coordinated activation of the immune system results in the mobilization of all major cellular subsets of innate and adaptive immunity within 2 weeks following immunization [13].

Similar to influenza vaccines, computational analysis has shown that gene signatures can predict magnitude of neutralizing antibodies, and models can be created capable of predicting high- and low$\mathrm{CD}^{+} \mathrm{T}$ cell responders to yellow fever vaccine [14]. 
Taken together, both influenza and yellow fever vaccines are potent inducers of genes associated with innate immunity and IFN responses, initiating a protective immune response. For both vaccines, several systems biology studies have recently been published correlating signatures to vaccine efficacy. These papers are discussed in more detail in other chapters of this issue (Dan Zak et al. / Bali Pulendran et al.).

\section{Major infectious diseases}

Despite enormous efforts, protective vaccines are still unavailable for the three major infectious diseases with highest global impact on public health: acquired immune deficiency syndrome (AIDS), malaria and tuberculosis (TB) [15-18].

One characteristic of the pathogens causing these diseases (human immunodeficiency virus or HIV, Plasmodium sp. and Mycobacterium tuberculosis, respectively) is the capability to evade or modulate immune defense responses. The mechanisms through which each pathogen achieves this, however, are radically different. HIV can establish a pool of dormant viruses inside macrophages [19]. During the active replication phase, HIV infects and destroys actively dividing T cells, targeting - among others those virus-specific T cells that fight the virus [20]. The malaria parasite displays high antigenic diversity to evade immune responses. Moreover, to ensure its survival it modulates DC maturation and function and even hijacks, and replicates within, DCs [21]. M. tuberculosis interferes with and blocks antibacterial phagocytic and apoptotic mechanisms of macrophages [22]. Overcoming such immune evasive mechanisms by these pathogens is one of the biggest challenges in vaccine research today.

In 2012, Wang et al [2] reviewed immunoprofiling studies in humans for vaccination against several diseases, including the three mentioned above.

In the search for an effective vaccine against HIV, numerous clinical trials have been, and are being, conducted (see http://aidsinfo.nih.gov/clinical-trials). Some of these employ transcriptional profiling using different candidate vaccine formulations. The prophylactic HIV lipopeptide candidate vaccine HIVLIPO-5 induces a high number of differentially expressed genes after vaccination, with a consistent overexpression of metallothionein genes [23]. The immune induction potency of this vaccine formulation is reflected by the transcriptional signatures associated with activation and motility of immune cells, differentiation and activation of T cells and differentiation of APCs. Similar to the vaccines described above, IFN signaling appears to be a major player in the response to HIV-LIPO-5.

Other approaches are targeted at developing therapeutic vaccines. Although the adaptive immune system is compromised in AIDS patients, the innate immune response induced by HIV virus-like particles (VLPs) is not reduced or abrogated in HIV-infected patients [24]. Furthermore, immune profiles appear qualitatively similar in high- and low-viral load patients. However, comparing transcriptional profiles in healthy seronegative individuals and HIV-infected people with high- or low-viral load can be daunting. Baseline expression profiles in the blood or unstimulated PBMCs differ significantly between uninfected and HIV-infected individuals, as shown, for example, by Monacoa et al [24]. This suggests that the distinct composition of PBMCs in healthy and HIV-infected individuals underlies these baseline differences, which in turn could contribute to reported baseline activation of chemokine genes in HIVinfected patients. 
Vaccine candidates against malaria have been tested for efficacy in healthy volunteers, where transcriptional signatures correlated with protective immune responses. Immunization with the adjuvanted RTS,S vaccine (sequences of the Plasmodium falciparum CSP expressed in hepatitis B surface antigen) induces distinct gene signatures in protected, nonprotected and partially protected individuals with delayed disease onset [25]. Protected and nonprotected study participants display different gene expression signatures associated with regulation of MHC processing in the immunoproteasome pathway. Similarly, genes associated with programmed cell death appear to be involved in protection against malaria blood-stage infection.

For the third major infectious disease, TB, a prophylactic vaccine has been in use since 1921. Mycobacterium bovis Bacille Calmette-Guérin (BCG) is administered to newborns in many endemic countries as vaccine against TB. Although it effectively prevents severe childhood disease, it does not protect against active pulmonary TB in all age groups. Transcriptomic analyses can have a profound impact on TB research [26-28]; yet, the number of high-throughput studies investigating the transcriptional responses to BCG vaccination in humans is surprisingly small. In experimental animal studies, BCG immunization of mice induces rapid transcriptional responses in the lung, which differ from those induced by live M. tuberculosis infection [29;30]. Early responses to BCG vaccination are dominated by IFN $\mathrm{Y}$-related genes, whereas after several weeks, increased expression in Th17 pathway-related genes is still detectable [29]. Transcriptome data can also identify in vitro predictors of protection upon BCG vaccination, as show in an M. bovis challenge model in cattle [31]. In this model cytokine-cytokine receptor interactions were found to play an important role in protective immune signatures. In humans, especially in BCG-vaccinated newborns, transcriptional blood signatures are usually the only available surrogate markers for pulmonary immune responses. Routine vaccination with BCG at birth induces upregulation of genes associated with the canonical pro-inflammatory macrophage response and changes in the cytokine-cytokine receptor pathway, while cell adhesion molecules appear to be downregulated [32]. Notably, a strong induction of genes involved in IFNY and IL-17 pathways can be observed in previously vaccinated populations [33], similar to responses observed in experimental mouse models [29]. Enhanced response signatures following booster vaccinations also reduce mycobacterial growth and increase scarring, correlating with previous vaccination. Whether an integrated combination of suppressed and upregulated genes can provide protective immunity against TB remains to be evaluated.

Intriguingly, different BCG vaccine strains used worldwide induce different immune responses, although no strain seems to provide superior protective capacity [34;35]. For example, gene expression signatures reveal significantly higher levels of cytokines characteristic of an adaptive immune response in neonates vaccinated with BCG-Moreau and BCG-Denmark strains, while BCG-Japan induces transcriptional signatures indicative of a higher proinflammatory response [36]. 


\section{Others}

Although numerous vaccines have been licensed and used in humans for some time, a comprehensive understanding of innate and adaptive immune responses to vaccination is still lacking. As shown in signatures discussed thus far, early innate immune processes are dominated by IFN-related and other pro-inflammatory responses.

Francisella tularensis is the etiological agent of tularemia [37]. The only currently available vaccine against human tularemia is the live vaccine strain (LVS), and the mechanisms of protection remain unclear. Most significant changes are induced during the first 2 days after immunization [38]. In addition to activation of innate immune processes, this vaccine induces strong modulation of antigen processing and presentation by DCs. Papillomavirus and smallpox vaccines induce similar inflammatory and IFN signatures [39;40], as well as cytokine production and genes associated with cytotoxicity and cell cycle pathways. Recent advances in measles vaccine immunogenetics are covered in detail by Haralambieva et al [41].

Not all individuals respond equally well to the same vaccine. For example, responsiveness in infants to hepatitis B vaccination can vary [42]. Altered methylation states of transcription factors and genes involved in antigen presentation may be involved in altered immune reactions related to poor responsiveness to vaccination.

A safe and efficacious vaccine against visceral leishmaniasis, which is fatal and endemic in many tropical countries, is still not available [43]. A review by Gannavaram et al provides an overview of vaccination strategies and biomarkers of safety and protection for genetically modified live attenuated Leishmania vaccine candidates [44].

\section{Boosting signatures}

Most infections cause a certain level of tissue damage and cell death, resulting in a "danger signal" that can trigger an immune response. Moreover, host immune cells sense pathogen-associated molecular patterns (PAMPs) through Toll-like receptors (TLRs) and other pattern recognition receptors (PRRs), thereby activating innate defense responses. Many vaccines mimic such signals by means of an adjuvant in the vaccine formulation [45]. Most advanced adjuvants, which are composed of surface-active components such as cationic peptides, saponins or polysorbates, include PAMPS like TLR9 ligands (oligodeoxynucleotides), TLR4 ligands (monophosphoryl lipid a, a low-toxic LPS derivative) and nucleotide-binding oligomerization domain (NOD)2-activating muramyl dipeptide for immune stimulation.

Other vaccines employ viral vectors for delivery of heterologous antigens. Attenuated vaccinia virus like modified vaccinia Ankara (MVA) is highly immunogenic, making it a valid candidate as vector expressing heterologous antigens from a wide spectrum of pathogens [46]. Attenuated poxvirus vectors MVA and NYVAC (New York attenuated vaccinia virus) induce similar responses in monocyte-derived DCs [47] although MVA induces an overall more potent inflammatory response profile. Genes involved in cytokine and chemokine signaling, molecules involved in antigen uptake and processing, as well as 
several transcription factors, are upregulated in response to both vectors. The downside of such in vitro studies is that only early time points can be analyzed due to the extensive cytopathic effects of these vectors. Illustrative of this are the transcriptional signatures induced by these vectors, which display a high percentage of genes functionally related to cell death [47]. Other vectors, such as adenoviruses, have been used for decades in vaccine development for their ability to express and deliver heterologous genes. While showing great potential in preclinical and clinical studies, several limitations affecting their in vivo efficacy still need to be overcome [48]. Adenovirus 5 (Ad5) induces differentiation of naïve antigen-specific CD8+ T cells into effector cells in the early phase and cells with memory phenotype at later stages [49]. Transcriptional profiles of these cells indicate a progressive up- or down-regulation of genes in different cell subsets following immunization with Ad5 [50]. Very similar transcriptional responses are also observed with recombinant lymphocytic choriomeningitis virus (rLCMV) as shown in the same study. Based on such transcriptional responses, antigen stimulation through these viral vectors appears to drive differentiation specifically from naïve, through central memory and effector memory towards effector CD8+ T cells [50].

Induction of effective immune responses often requires prolonged release of antigen. These depot effects can be induced by emulsions of oil in water or by liposomes. Incorporation of CpG (a TLR9 ligand) into liposomes drives IFN responses, with a highly enriched signature for anti-viral and IFN-mediated immunity in afferent lymph cells [51]. These signatures are most prominent in lymphocytes, and could help in sustaining the vaccine-induced immune response at the site of injection.

Most widely used in licensed vaccines are aluminum salts (like alum), while experimental preclinical vaccine studies frequently employ oil-in-water emulsions [52]. Most adjuvants promote the recruitment of APCs into the injection site and the modulation a shared set of genes. Adjuvant administration of MF59 (oil-in-water formulation), alum and a nonmethylated $\mathrm{CpG}$ oligonucleotide all induce rapid upregulation of genes involved in cytokine-cytokine receptor interactions, as well as genes involved in antigen processing and presentation [53]. The use of oil-in-water emulsions like in MF59 induces an early and strong immune-competent environment, which might make it a highly efficient vaccine adjuvant for humans. However, until we understand what an efficacious response should look like, other options cannot be excluded. Vaccine preparations against different pathogens will likely also require distinct pathogen-congruent adjuvant formulations to induce the desired response. Although MF59 induces a more prominent and broader local response in the muscle of experimental mice [53], it needs to be verified whether it induces the same type of response in humans.

Next-generation adjuvants confer proinflammatory effects through TLR signaling, which induces a potent response mimicking infection with a viable pathogen. Based on evidence that many vaccines induce protective $\mathrm{T}$ - and $\mathrm{B}$-cell responses through the innate immune system, Kwissa et al analyzed several TLR ligands for their potency to induce innate responses in rhesus macaques [54]. A rapid expansion of blood neutrophils and distinct monocyte subtypes was induced by all three ligands, MPL (TLR4 ligand), R-848 (TLR7/8 ligand) and CpG (TLR9 ligand). Remarkably, the typically dominant IFNrelated genes were not induced by the TLR4 ligand, in contrast to TLR7/8 and TLR9 ligands. Further differences were rapidly increased levels of inflammatory cytokines in the blood after stimulation with 
342 the TLR7/8 ligand, compared to a delayed and more modest increase in inflammatory cytokines with the 343 TLR9 ligand.

344 Thus, adjuvant formulations targeting different TLR signaling routes trigger distinct transcriptional and 345 cellular signatures.

346

347

\section{Conclusion}

349 It is encouraging that the field of vaccinology has adopted high-throughput profiling techniques to gain a 350 more detailed understanding of immune responses elicited by different vaccines. Without doubt, this 351 knowledge will facilitate the design of next-generation vaccine candidates in the future. Innate immune 352 responses are not only a first defense mechanism against invading pathogens but are also crucial for the 353 development of subsequent protective adaptive immune responses. This relatively recent insight has 354 brought about a shift in attention towards early molecular signatures in high-throughput vaccine 355 studies. Detailed transcriptional profiles induced by a variety of vaccines are dominated by IFN 356 signatures. But simultaneously, vaccines against various types of pathogens induce distinct gene 357 expression profiles characteristic of pathogen-specific defense responses. Further research of immune 358 signatures - correlated with vaccine efficacy and safety - is needed to custom design vaccine 359 formulations for the plethora of pathogens which cause major health threats. 


\section{Acknowledgements}

362 The authors thank Mary Louise Grossman for help preparing the manuscript. Work in the authors' lab is

363 supported by the European Union's Seventh Framework Programme (EU FP7) project "ADITEC" 364 (HEALTH-F4-2011-280873; and EU Horizon 2020 project "TBVAC 2020" (grant 643381); the Innovative 365 Medicines Initiative (IMI) Joint Undertaking (JU) "Biomarkers for Enhanced Vaccine Safety" project 366 BioVacSafe (IMI JU Grant No. 115308); the Bill \& Melinda Gates Foundation (BMGF) "Grand Challenges 367 (GC) in Global Health" Program (BMGF GC6-2013, (\#OPP 1055806 and \#OPP 1065330); and the 368 Bundesministerium für Bildung und Forschung (BMBF) project "Infect Control 2020."

369

370 The authors declare no conflicts of interest.

371

372 


\section{Box 1. Key components of host defense mechanisms}

Antigen presenting cells (APCs)

Cells that process and display antigens to T lymphocytes

Dendritic cells (DCs)

Professional APCs; several specialized subtypes exist in blood and in tissue exposed to external environment

Natural killer cells (NKs)

Cytotoxic lymphocytes, part of the innate immune system; provide rapid responses to virusinfected cells

\section{Macrophages}

Central role in non-specific defense responses; engulf and digest/destroy cellular debris, microbes and aberrant cells

\section{Neutrophils}

Phagocytic cells involved in first line of defense against invading pathogens; highly abundant in blood, recruited to site of inflammation

\section{T lymphocytes/T cells}

Cells of the adaptive immune system; play a central role in cell-mediated immunity; regulating immune responses and mediating destruction of infected cells

\section{B lymphocytes/B cells}

Cells of the adaptive immune system; play a central role in humoral immunity by producing antigen-specific antibodies; can act as APCS

Pattern recognition receptors (PRRs)

Recognize molecules associated with pathogens; activate innate immune responses

Toll-like receptors (TLRs)

A subgroup of pathogen-associated molecular patterns (PAMPs); recognize structurally conserved molecules from pathogens; initiate innate immune responses

\section{Chemokines}

Signaling proteins secreted by cells; mediate chemotactic signals to cells of the immune system

\section{Cytokines} to migrate into defined sites, e.g., inflammatory foci

Signaling proteins secreted by cells; regulate immune responses, maturation and activation of specific cell populations

Interleukins (ILS) Cytokines typically produced by, and acting on, leukocytes; used for systematic nomenclature (IL-1, IL-2, etc.)

Interferon (INF)

Type I IFN: central cytokine of the innate defense mechanism; secreted by cells in response to pathogens; type II IFN: primarily produced by T cells; both IFNs trigger several signaling pathways leading to a typical IFN gene expression signature

Inflammasome

Component of the innate immune system; protein complex promoting maturation of certain inflammatory cytokines, notably IL-1ß and IL-12 
[1] Flanagan KL, Noho-Konteh F, Ghazal P, Dickinson P. Transcriptional profiling technology for studying vaccine responses: an untapped goldmine. Methods 2013 May 1;60(3):269-74.

[2] Wang IM, Bett AJ, Cristescu R, Loboda A, ter MJ. Transcriptional profiling of vaccine-induced immune responses in humans and non-human primates. Microb Biotechnol 2012 Mar;5(2):177-87.

[3] Jenner RG, Young RA. Insights into host responses against pathogens from transcriptional profiling. Nat Rev Microbiol 2005 Apr;3(4):281-94.

[4] Banchereau R, Baldwin N, Cepika AM, et al. Transcriptional specialization of human dendritic cell subsets in response to microbial vaccines. Nat Commun 2014;5:5283.

[5] WHO. Vaccines against influenza WHO position paper - November 2012. Wkly Epidemiol Rec 2015 Nov 23;87(47):461-76.

[6] Gomez Lorenzo MM, Fenton MJ. Immunobiology of influenza vaccines. Chest 2013 Feb 1;143(2):502-10.

[7] Zhu W, Higgs BW, Morehouse C, et al. A whole genome transcriptional analysis of the early immune response induced by live attenuated and inactivated influenza vaccines in young children. Vaccine 2010 Apr 1;28(16):2865-76.

[8] Bucasas KL, Franco LM, Shaw CA, et al. Early patterns of gene expression correlate with the humoral immune response to influenza vaccination in humans. J Infect Dis 2011 Apr 1;203(7):9219.

[9] Nakaya HI, Wrammert J, Lee EK, et al. Systems biology of vaccination for seasonal influenza in

[10] Li S, Rouphael N, Duraisingham S, et al. Molecular signatures of antibody responses derived from a systems biology study of five human vaccines. Nat Immunol 2014 Feb;15(2):195-204.

[11] Obermoser G, Presnell S, Domico K, et al. Systems scale interactive exploration reveals quantitative and qualitative differences in response to influenza and pneumococcal vaccines. Immunity 2013 Apr 18;38(4):831-44.

[12] WHO. Yellow fever. Fact sheet Nº100 March 2014. WHO Media Centre 2014.

[13] Gaucher D, Therrien R, Kettaf N, et al. Yellow fever vaccine induces integrated multilineage and polyfunctional immune responses. J Exp Med 2008 Dec 22;205(13):3119-31.

[14] Querec TD, Akondy RS, Lee EK, et al. Systems biology approach predicts immunogenicity of the yellow fever vaccine in humans. Nat Immunol 2009 Jan;10(1):116-25. 
[15] Karunamoorthi K. Malaria vaccine: a future hope to curtail the global malaria burden. Int J Prev Med 2014 May;5(5):529-38.

[16] Delva W, Abdool KQ. The HIV epidemic in Southern Africa - Is an AIDS-free generation possible? Curr HIV /AIDS Rep 2014 Jun;11(2):99-108.

[17] Gengenbacher M, Kaufmann SH. Mycobacterium tuberculosis: success through dormancy. FEMS Microbiol Rev 2012 May;36(3):514-32.

[18] Kaufmann SH, McElrath MJ, Lewis DJ, Del GG. Challenges and responses in human vaccine development. Curr Opin Immunol 2014 Jun;28:18-26.

[19] Archin NM, Sung JM, Garrido C, Soriano-Sarabia N, Margolis DM. Eradicating HIV-1 infection: seeking to clear a persistent pathogen. Nat Rev Microbiol 2014 Nov;12(11):750-64.

[20] Ipp $\mathrm{H}$, Zemlin A. The paradox of the immune response in HIV infection: when inflammation becomes harmful. Clin Chim Acta 2013 Feb 1;416:96-9.

[21] Stanisic DI, Barry AE, Good MF. Escaping the immune system: How the malaria parasite makes vaccine development a challenge. Trends Parasitol 2013 Dec;29(12):612-22.

[22] Cambier CJ, Falkow S, Ramakrishnan L. Host Evasion and Exploitation Schemes of Mycobacterium tuberculosis. Cell 2014 Dec 18;159(7):1497-509.

[23] Richert L, Hue $\mathrm{S}$, Hocini $\mathrm{H}$, et al. Cytokine and gene transcription profiles of immune responses elicited by HIV lipopeptide vaccine in HIV-negative volunteers. AIDS 2013 Jun 1;27(9):1421-31.

[24] Monaco A, Marincola FM, Sabatino M, et al. Molecular immune signatures of HIV-1 vaccines in human PBMCs. FEBS Lett 2009 Sep 17;583(18):3004-8.

[25] Vahey MT, Wang Z, Kester KE, et al. Expression of genes associated with immunoproteasome processing of major histocompatibility complex peptides is indicative of protection with adjuvanted RTS,S malaria vaccine. J Infect Dis 2010 Feb 15;201(4):580-9.

[26] Zarate-Blades CR, Silva CL, Passos GA. The impact of transcriptomics on the fight against tuberculosis: focus on biomarkers, BCG vaccination, and immunotherapy. Clin Dev Immunol 2011;2011:192630.

[27] Maertzdorf J, Weiner J, III, Kaufmann SH. Enabling biomarkers for tuberculosis control. Int J Tuberc Lung Dis 2012 Sep;16(9):1140-8.

[28] Weiner J, Maertzdorf J, Kaufmann SH. The dual role of biomarkers for understanding basic principles and devising novel intervention strategies in tuberculosis. Ann N Y Acad Sci 2013 Apr;1283:22-9.

[29] Aranday CE, Kaveh D, Nunez-Garcia J, Hogarth PJ, Vordermeier HM. Mycobacterium bovis-BCG vaccination induces specific pulmonary transcriptome biosignatures in mice. PLoS One 2010;5(6):e11319. 
[30] Mollenkopf HJ, Hahnke K, Kaufmann SH. Transcriptional responses in mouse lungs induced by vaccination with Mycobacterium bovis BCG and infection with Mycobacterium tuberculosis. Microbes Infect 2006 Jan;8(1):136-44.

[31] Bhuju S, Aranday-Cortes E, Villarreal-Ramos B, Xing Z, Singh M, Vordermeier HM. Global gene transcriptome analysis in vaccinated cattle revealed a dominant role of IL-22 for protection against bovine tuberculosis. PLoS Pathog 2012 Dec;8(12):e1003077.

[32] Fletcher HA, Keyser A, Bowmaker M, et al. Transcriptional profiling of mycobacterial antigeninduced responses in infants vaccinated with BCG at birth. BMC Med Genomics 2009;2:10.

[33] Matsumiya M, Satti I, Chomka A, et al. Gene Expression and Cytokine Profile Correlate With Mycobacterial Growth in a Human BCG Challenge Model. J Infect Dis 2014 Nov 7.

[34] Ritz N, Hanekom WA, Robins-Browne R, Britton WJ, Curtis N. Influence of BCG vaccine strain on the immune response and protection against tuberculosis. FEMS Microbiol Rev 2008 Aug;32(5):821-41.

[35] Ritz N, Dutta B, Donath S, et al. The influence of bacille Calmette-Guerin vaccine strain on the immune response against tuberculosis: a randomized trial. Am J Respir Crit Care Med 2012 Jan 15;185(2):213-22.

[36] Wu B, Huang C, Garcia L, et al. Unique gene expression profiles in infants vaccinated with different strains of Mycobacterium bovis bacille Calmette-Guerin. Infect Immun 2007 Jul;75(7):3658-64.

[37] Carvalho CL, Lopes dC, I, Ze-Ze L, Nuncio MS, Duarte EL. Tularaemia: a challenging zoonosis. Comp Immunol Microbiol Infect Dis 2014 Mar;37(2):85-96.

[38] Fuller CL, Brittingham KC, Porter MW, et al. Transcriptome analysis of human immune responses following live vaccine strain (LVS) Francisella tularensis vaccination. Mol Immunol 2007 May;44(12):3173-84.

[39] Garcia-Pineres AJ, Hildesheim A, Dodd L, et al. Gene expression patterns induced by HPV-16 L1 virus-like particles in leukocytes from vaccine recipients. J Immunol 2009 Feb 1;182(3):1706-29.

[40] Haralambieva IH, Oberg AL, Dhiman N, et al. High-dimensional gene expression profiling studies in high and low responders to primary smallpox vaccination. J Infect Dis 2012 Nov 15;206(10):151220.

[41] Haralambieva IH, Ovsyannikova IG, Pankratz VS, Kennedy RB, Jacobson RM, Poland GA. The genetic basis for interindividual immune response variation to measles vaccine: new understanding and new vaccine approaches. Expert Rev Vaccines 2013 Jan;12(1):57-70.

[42] Lu Y, Cheng Y, Yan W, Nardini C. Exploring the molecular causes of hepatitis B virus vaccination response: an approach with epigenomic and transcriptomic data. BMC Med Genomics 2014;7:12.

[43] Kumar R, Engwerda C. Vaccines to prevent leishmaniasis. Clin Transl Immunology 2014 Mar;3(3):e13. 
[44] Gannavaram S, Dey R, Avishek K, Selvapandiyan A, Salotra P, Nakhasi HL. Biomarkers of safety and immune protection for genetically modified live attenuated leishmania vaccines against visceral leishmaniasis - discovery and implications. Front Immunol 2014;5:241.

[45] Maisonneuve C, Bertholet S, Philpott DJ, De GE. Unleashing the potential of NOD- and Toll-like agonists as vaccine adjuvants. Proc Natl Acad Sci U S A 2014 Aug 26;111(34):12294-9.

[46] Moss B. Genetically engineered poxviruses for recombinant gene expression, vaccination, and safety. Proc Natl Acad Sci U S A 1996 Oct 15;93(21):11341-8.

[47] Guerra S, Najera JL, Gonzalez JM, et al. Distinct gene expression profiling after infection of immature human monocyte-derived dendritic cells by the attenuated poxvirus vectors MVA and NYVAC. J Virol 2007 Aug;81(16):8707-21.

[48] Appaiahgari MB, Vrati S. Adenoviruses as gene/vaccine delivery vectors: promises and pitfalls. Expert Opin Biol Ther 2015 Mar;15(3):337-51.

[49] Opferman JT, Ober BT, Ashton-Rickardt PG. Linear differentiation of cytotoxic effectors into memory T lymphocytes. Science 1999 Mar 12;283(5408):1745-8.

[50] Roychoudhuri R, Lefebvre F, Honda M, et al. Transcriptional profiles reveal a stepwise developmental program of memory CD8(+) T cell differentiation. Vaccine 2015 Feb 11;33(7):91423.

[51] Neeland MR, Elhay MJ, Powell DR, Rossello FJ, Meeusen EN, de Veer MJ. Transcriptional profile in afferent lymph cells following vaccination with liposomes incorporating CpG. Immunology 2014 Oct 10.

[52] Schijns VE, Strioga M, Ascarateil S. Oil-based emulsion vaccine adjuvants. Curr Protoc Immunol 2014;106:2-7.

[53] Mosca F, Tritto E, Muzzi A, et al. Molecular and cellular signatures of human vaccine adjuvants. Proc Natl Acad Sci U S A 2008 Jul 29;105(30):10501-6.

[54] Kwissa M, Nakaya HI, Oluoch H, Pulendran B. Distinct TLR adjuvants differentially stimulate systemic and local innate immune responses in nonhuman primates. Blood $2012 \mathrm{Mar}$ 1;119(9):2044-55. 